\title{
Understanding Within-Session Loss-Chasing: An Experimental Investigation of the Impact of Stake Size on Cognitive Control
}

\author{
Adrian Parke ${ }^{1}$ - Andrew Harris ${ }^{1}$ Jonathan Parke ${ }^{2}$. \\ Paul Goddard ${ }^{1}$
}

(C) Springer Science+Business Media New York 2015

\begin{abstract}
Loss-chasing is a central feature of problematic gambling, yet it remains a poorly conceived and understood concept. Loss-chasing is believed to stem from an erosion of cognitive control when gambling. The opportunity to gamble at significantly disparate stake sizes on a gambling activity is considered to be a risk factor for loss-chasing. This study investigated the impact of gambling at disparate stake sizes on executive processes integral to maintaining cognitive control when gambling, namely response inhibition and reflection impulsivity. Frequent adult non-problem gamblers $(n=32)$ participated in a repeated measures experiment; and gambled at three disparate stake sizes ( $£ 20, £ 2$ and no stake per bet) on a simulated gambling task. Participants' response inhibition performance and reflection impulsivity levels after gambling at various stake sizes were compared via a go/no-go task and information sampling task, respectively. Quality of decision-making i.e. the evaluation of available information to make probability judgements was impaired after gambling at higher stakes in comparison to lower stakes, indicating an increase in reflection impulsivity. No effect on response inhibition was observed. Although exploratory, this suggests that the opportunity for participants to substantially increase stake size on a gambling activity may be a risk factor for impaired cognitive performance when gambling, and perhaps create vulnerability for within-session loss-chasing in some players.
\end{abstract}

Keywords Problem gambling · Cognitive control - Loss-chasing · Response inhibition · Reflection impulsivity

Adrian Parke

aparke@lincoln.ac.uk

1 School of Psychology, University of Lincoln, Brayford Campus, Lincoln LN6 8AP, UK

2 Psychology Department, University of Bangor, Bangor, UK 


\section{Introduction}

\section{Loss Chasing: A Central Feature of Problem Gambling}

The chasing of one's gambling losses is a frequently observed pattern of gambling behaviour in both social gamblers (Dickerson et al. 1987) and problem gamblers (DSMIV-TR, American Psychiatric Association 2000). Fundamentally, it is widely accepted that chasing of losses when gambling is the most significant step towards developing a gambling disorder (Breen and Zuckerman 1999; Goudriaan et al. 2014; Lesiuer 1979). It is argued that extended loss-chasing can precipitate a rapid downward spiral of gambling involvement for the individual, where liabilities increase to such an extent that there are significant negative and deleterious consequences for the individual, including familial, social and occupational costs (Corless and Dickerson 1989; Sharpe 2002). However, despite the centrality of loss-chasing to the development of gambling disorders, very little is understood about the phenomena (Campbell-Meiklejohn et al. 2008).

It is proposed that before understanding the psychological mechanism underlying losschasing over an extended time period (between-session), it is first more prudent to begin to understand within-session chasing where the loss of control of gambling behaviour is limited to a single gambling session (Breen and Zuckerman 1999; O'Connor and Dickerson 2003).

Although many etiological models of gambling disorder identify erroneous cognitive heuristics as a central feature (e.g. Blaszczynski and Nower 2002), Potenza (2014) concluded that irrational cognitions are unlikely to be the primary cause of loss of control in gambling. However, it is evident there is a significant erosion of cognitive control regarding the urge to persist in gambling despite the overt adverse consequences being experienced (Goudriaan et al. 2014). Goldstein and Volkow (2002) characterise this persistence in gambling despite the negative consequences as a deficit in self-regulation. Selfregulatory deficits in individuals with addiction disorders are understood to generate through dysregulation in the primitive brain structures that process reward, and after repeated exposure, this deficit affects more complex executive functioning (Kräplin et al. 2014; Volkow et al. 2010).

\section{Understanding Within-Session Loss of Cognitive Control}

It is proposed that executive functioning during a gambling session will monitor, organise and bias lower level cognitive processes, and respond appropriately to feedback from the game and the environment, in order to meet pre-determined, or updated gambling goals. van Holst et al. (2010) concluded that while it is clearly evident, from reviewing behavioural decision-making research literature, that problem gamblers often have less efficient executive functioning than healthy control populations, it is not currently understood what the underlying processes are that support such disadvantageous behavioural choices. The executive control deficit observed in problem gamblers has often been characterised as impulsivity, but at a neuropsychological level, impulsivity is understood to stem from deficits in inhibitory control (Lawrence et al. 2009a). Rather than a singular construct such as impulsivity, it is probable that the loss of cognitive control, observed in within-session chasing, is a result of multiple contributing sub-processes (Goudriaan et al. 2014), and that investigation of individual lower order decision-making components is likely to be the most effective approach to understanding the dysfunction (Kräplin et al. 2014). 
When attempting to understand if specific executive control processes contribute to within-session loss-chasing behaviour it is prudent to begin exploring two of the most commonly observed executive function deficits observed in addiction populations, including problem gamblers, principally Response Inhibition (Billieux et al. 2012; Conversano et al. 2012; Goudriaan et al. 2006; Kertzman et al. 2008) and Reflection Impulsivity (Lawrence et al. 2009b; Clark et al. 2009a; Cohen et al. 2010; Quednow et al. 2007).

Specifically, response inhibition could be conceptualised as the process of not attenuating to a strongly conditioned cue (Wright et al. 2014). Poor response inhibition performance, often referred to as 'behavioural disinhibition', demonstrates an inability to restrain a response that is no longer adaptive, or at least no longer appropriate (Strakowski et al. 2009; Winstanley et al. 2006). Barkley (1997) argues that three interrelated responses are involved including inhibiting a prepotent response, and thus allowing a delay responding, and finally the protection of this delay from competing signals, i.e. resisting interference.

Alternatively, reflection impulsivity refers to the tendency to make an impulsive decision in contrast to the tendency to gather and evaluate information prior to decisionmaking. In reference to adult psychopathology, more weighting has been given to the dependent outcome of accuracy of decision-making in response to available information rather than being centrally focused upon the latency of decisions (Block et al. 1974; Clark et al. 2006). Higher reflection impulsivity leads to poorly conceived behaviour that is prematurely expressed, and inappropriately risky in response to a specific situation (Clark et al. 2009b).

Given the most consistently observed cognitive deficits in problem gamblers relate to processes within the ventromedial prefrontal cortex (vmPFC) and/or low-level risk and reward decision-making (Potenza 2014), the ability to inhibit automatic responses to reward and withstand such interference to make advantageous behavioural decisions appears central to avoiding within-session loss-chasing. Furthermore, the facilitation of attentional control through effective response inhibition (Derryberry and Reed 2002) is likely to enable the individual to modulate reflexive emotional responses to gambling outcomes and subsequently facilitate advantageous behavioural decisions (Spada and Roarty 2015). Within the context of within-session loss-chasing, response inhibition appears fundamental to suppressing dominant responses to arousing stimulation when incurring gambling losses; and when doing so, is likely to facilitate the individual in tempering negative emotional responses to losses and potentially reducing reflection impulsivity.

\section{Size of Stake and Within-Session Loss of Cognitive Control}

In the literature exploring the explanatory role of executive control dysfunction in problem gambling, there is an expectation that such deficits predate the gambling disorder, and therefore are pre-existing individual vulnerabilities to developing a gambling disorder (Belin et al. 2008; Verdejo-Garcia et al. 2008). It therefore follows that if within-session chasing of gambling losses can also be explained through individual deficits in cognitive control, one would conceive the deficit to be a pre-existing vulnerability. However, structural characteristics of the gambling activity are also crucial determinants of arousal and reinforcement in the development of disordered gambling patterns (Blaszczynski 2013). Research has demonstrated that structural features, such as speed of game (Delfabbro et al. 2005), schedule of reinforcement volatility (Coates and Blaszczynski 2013; Dixon et al. 2006), maximum bet (Livingstone et al. 2008), and near-misses (Clark et al. 2009a; Clark et al. 2012), affect the gambler's willingness to continue gambling 
despite incurring significant losses (Blaszczynski 2013). It is possible that such structural characteristics can influence executive control in the individual in the short-term, including response inhibition and reflection impulsivity, and therefore potentially explain withinsession loss of cognitive control.

Gambling at higher and variable staking size is predictive of disordered gambling (Braverman and Shaffer 2010; Sharpe et al. 2005). Ostensibly, the amount that can be lost within a gambling session is determined through four primary factors: the speed per event, the percentage return, game volatility and size of stake (Parke and Parke 2013). Of the four structural characteristics that determine cost of play, size of stake is the variable that gamblers can most readily change. Along with persistent gambling in the face of incurring significant losses, the escalation of bet size is considered to be a central feature of losschasing (Walker 1992). Therefore, investigating the short-term impact of betting at different stake sizes on response inhibition and reflection impulsivity may be a useful first step in trying to explain within-session loss-chasing in terms of a temporary reduction in cognitive control.

\section{Research Aims}

It is widely accepted that specific executive processes such as response inhibition and reflection impulsivity can be observed within laboratory settings (Holmes et al. 2014; Lawrence et al. 2009b), including changes in performance and efficiency (Stevens et al. 2015). The aim of the current study is to begin to understand the individual contribution of size of stake on cognitive control when gambling, specifically focussing upon the immediate impact on response inhibition and reflection impulsivity.

\section{Research Hypotheses}

- Response inhibition performance, immediately after the gambling event, will be reduced in the high stake condition in comparison to gambling in the low stake and no stake conditions.

- Reflection impulsivity will be higher after gambling in the high stake condition in comparison to gambling in the low stake and no stake conditions.

\section{Method}

\section{Participants}

A sample of 32 adults (30 male), who were self-reported non-problem gamblers were recruited via a series of online advertisements posted within local interest, gambling and sporting web-communities that were geographically proximal to the university. The inclusion criteria for the study included recent experience of virtual roulette, so that the gambling task in the experiment was not a novelty; and that they were frequent gamblers, defined as gambling at least once every 14 days. Information regarding participants' regular gambling patterns was obtained via brief semi-structured interviews prior to engagement in the experimental tasks. Participants were asked if they had ever selfexcluded from a gambling environment, either online or offline, or if they had experienced social problems because of gambling, or finally, had sought help regarding problems 
related to gambling. If a participant had answered positively to the questions related to problem gambling, they would have been assessed with the Problem Gambling Severity Index (Ferris and Wynne 2001) and would have been subsequently excluded from the study if they indeed met the criteria for problem gambling. None of the 32 participants answered positively to the problem gambling questions within the pre-experiment interviews.

\section{Measures}

\section{Go/No-Go Task}

Response inhibition performance was recorded via a modified version of the classical go/ no-go task (Fillmore 2003), which is a measure of ability to withhold a prepotent response as well as attention, vigilance and reaction time (Wright et al. 2014). Participants were presented with directional arrows, pointing either left or right, on a computer screen, for a period of $1500 \mathrm{~ms}$, with each arrow cued by a fixation point for a duration of $500 \mathrm{~ms}$. Participants were requested to, as quickly as possible, respond by pressing the corresponding left or right arrow key on a QWERTY keyboard to the arrow presented onscreen. After developing a prepotent response, participants were informed that when an arrow was simultaneously presented with a 'beep' sound effect (150 $\mathrm{ms}$ in duration) to withhold from pressing the arrow key on the keyboard. Go trials consisted of the singular directional arrow and stop trials consisted of the arrow and the audio beep.

The mean percentage of commission errors, where participants did not withhold the prepotent response, was recorded as measure of response inhibition performance. In addition, the mean percentage of omission trials, where participants failed to respond correctly to the directional arrow presented, and mean response time were also measured, as indicators of attention and vigilance respectively (Wright et al. 2014).

\section{Information Sampling Task (IST)}

Reflection impulsivity was measured using a modified version of the information sampling task developed by Clark et al. (2006). The original IST consisted of a grid of $5 \times 5$ covered boxes that were one of two unseen colours, and participants were required to sequentially select a box to reveal the colour underneath the cover. Participants were required to identify which of the two colours would be in the majority when all boxes were uncovered (see Clark et al. 2006).

In the current study, the IST was modified to be more representative of a familiar probability task, and was based upon the classic Urn Problem. The urn contained 19 unseen balls, and each ball was coloured black or red. Participants were required to determine whether red or black balls were in the majority. Participants were able to gather information by selecting to remove a ball from the urn to reveal its colour. Points were awarded in the IST for correct predictions regarding which colour was in the majority. For each IST trial participants began with 95 points, and had 5 points removed for each ball taken from the urn. For example, if a participant removed 3 balls from the urn, and correctly predicted that red was in the majority they would win 80 points for that IST trial. However, if participants made an incorrect prediction, then they would receive 0 points for that trial. Participants were informed that all of the points that they accumulated over the IST trials would be totalled, and the participant who accumulated the most points would 
receive a $£ 100$ cash prize. During the experiment, participants were not provided with feedback regarding whether their predictions in each IST trial were correct.

Reflection impulsivity was measured by observing three component variables: Mean Information Sampled, Mean Response Latency and Mean Probability of Making Correct Decisions (Pcorrect). Mean Information Sampled referred to the average number of balls removed from the urn for each IST trial, and the Mean Response Latency refers to the average amount of time, used to make a decision to remove another ball or to make a prediction of which colour was in the majority. Mean Probability of Making Correct Decisions (Pcorrect) referred the probability of the participants' colour predictions being accurate, based on the available information at the time the decision was made (Clark et al. 2006).

\section{Design and Procedure}

After a detailed instructional session, where participants were informed about the task order and requirements, and also provided within an opportunity to engage in practice IST and go/no-go tasks to ensure comprehension, participants provided informed written consent (Ethical Application Approval Number: PSY131404). Participants were then provided with casino chips (worth a total of £132) that they were required to bet in the various high and low stake gambling conditions, and informed they were allowed to keep any winnings and stakes on trials where their bets won, and that any money staked on losing bets would be permanently lost.

In the gambling task participants were presented with a simplified virtual roulette task, where the participant was required to risk either $£ 20$ (High Stake Condition), $£ 2$ (Low Stake Condition) or no stake (Control Condition), in a 1:1 bet whether the marble would land on a red or black section of the roulette wheel. Participants were informed that the outcome of the virtual roulette trials was determined by random chance.

The experiment consisted of a repeated-measures design, where each participant undertook the gambling task, the go/no-go and the IST in all five experimental conditions, including: high stake winning, high stake losing, low stake winning, low stake losing and the no stake control condition. In each condition participants were required to make three separate bets (three gambling task trials) on virtual roulette, at the prescribed bet size. After the participant bet whether the marble would land on either red or black, the virtual roulette wheel would spin for $13,000 \mathrm{~ms}$, and the game would indicate via onscreen text whether the participant had won ('Winner') or lost ('You Lost'). Immediately after the result of the bet was revealed the experimenter would either remove the lost stake or provide the participant with the money that was won (in the form of casino chips).

After each virtual roulette bet, the participant would complete the go/no-go-task to measure response inhibition performance. In each condition, participants would complete the gambling task followed by the go/no-go task three times. After the three virtual roulette bets and go/no-go tasks, the participants were provided with an onscreen reminder of how much money they had won or lost in that condition. After observing the reminder of incurred wins or losses in the betting trials the participant would be required to complete 5 trials of the information sampling task to assess reflection impulsivity level.

In both the high stake and low stake winning conditions participants won each of their three gambling task trials, and equally, in both losing conditions the participants lost each of the three bets. The order each participant would experience the conditions was counterbalanced to minimise order-effect. Participants were provided with a 15 min rest period between each experimental condition. 


\section{Data Analysis}

Independent variables of the study included size of stake (£20, £2 or $£ 0)$. Dependent variables of the information sampling task study included Mean Information Sampled, Mean Response Latency and Mean Probability of Making Correct Decisions (Pcorrect). Dependent variables of the go/no-go task included: Mean Percentage of Commission Errors, Mean Percentage of Omission Errors and Mean Response Time. Data for the information sampling task and the go/no-go task were evaluated using repeated-measures ANOVA, using Greenhouse-Geisser epsilon where sphrecity assumptions were violated. Between group comparisons were investigated using Bonferroni's correction.

\section{Results}

\section{The Effect of Stake Size on Post-gambling Response Inhibition}

The mean amount of commission errors was higher in the high stake condition in comparison to the low stake condition; however this difference in commission errors was not statistically significant: $\mathrm{F}(2,62)=1.48, p>0.05$. Furthermore, the mean amount of omission errors, an indication of participant attention and vigilance, was also higher in the high stake condition in comparison to the low stake and control conditions, but again this difference was not statistically significant: $F(2,62)=0.986, p>0.05$. Finally, there was no statistically significant difference in mean reaction time across the three stake size conditions: $F(2,62)=1.978, p>0.05$ (Table 1 ).

When contrasting the mean commission error rates, there were no statistically significant differences between stake size conditions whether the participants won bets or lost bets. Equally, there were no statistically significant differences in mean omission error rates or mean reaction times across stake size conditions, whether participants were in winning or losing experimental conditions.

\section{Reflection Impulsivity}

\section{Information Sampled}

There was a statistically significant difference between the mean amount of information sampled (the mean number of balls removed from the urn) across betting at different stake sizes: $F(1.64,50.854)=3.882, p<0.05$, partial $\eta^{2}=0.111$. However, post hoc analysis showed that there was only a significant difference in mean amount of balls removed between the high stake and control conditions $(p=0.012)$. Similarly, although there was statistically significant difference in mean amount of information sampled between losing high stake, losing low stake and control conditions $(F(2,62)=5.652, p<0.05$, partial $\left.\eta^{2}=0.154\right)$, there was only a significant difference between the high stake and the control conditions $(p=0.004)$. There was no statistically significant difference in mean amount of information sampled between winning high stake, winning low stake and control conditions: $F(2,62)=1.432, p>0.05$ (Table 2$)$. 
Table 1 Means and standard deviations of response inhibition performance components after gambling at various stake sizes and betting outcomes

\begin{tabular}{llll}
\hline Stake size & $\begin{array}{l}\text { Mean commission } \\
\text { errors (SD) }\end{array}$ & $\begin{array}{l}\text { Mean omission } \\
\text { errors (SD) }\end{array}$ & $\begin{array}{l}\text { Mean reaction time } \\
\text { in ms (SD) }\end{array}$ \\
\hline Control (£0 per bet) & $9.17(7.48)$ & $2.90(2.75)$ & $369.6(35.8)$ \\
Low stake (£2 per bet) & $9.52(6.09)$ & $2.91(2.69)$ & $364.9(35.7)$ \\
High stake (£20 per bet) & $10.77(8.9)$ & $3.24(3.04)$ & $364.7(36.0)$ \\
Low stake losing & $8.97(6.34)$ & $2.58(2.44)$ & $365.7(35.1)$ \\
High stake losing & $10.49(8.78)$ & $3.18(3.28)$ & $364.1(39.4)$ \\
Low stake winning & $10.08(8.10)$ & $3.23(3.10)$ & $364.2(37.0)$ \\
High stake winning & $11.04(10.17)$ & $3.29(3.09)$ & $367.8(37.7)$ \\
\hline
\end{tabular}

Table 2 Means and standard deviations of reflection impulsivity performance components after gambling at various stake sizes and betting outcomes

\begin{tabular}{llll}
\hline Stake size & $\begin{array}{l}\text { Mean amount of } \\
\text { information sampled (SD) }\end{array}$ & $\begin{array}{l}\text { Mean response } \\
\text { latency in s (SD) }\end{array}$ & $\begin{array}{l}\text { Probability of making } \\
\text { correct prediction (SD) }\end{array}$ \\
\hline Control (£0 per bet) & $3.71(2.1)$ & $2.32(0.8)$ & $0.629(0.07)$ \\
Low stake (£2 per bet) & $3.73(2.0)$ & $2.64(2.0)$ & $0.614(0.08)$ \\
High stake (£20 per bet) & $3.11(2.1)$ & $3.36(3.3)$ & $0.591(0.08)$ \\
Low stake losing & $3.38(2.1)$ & $3.01(2.7)$ & $0.608(0.09$ \\
High stake losing & $2.88(2.2)$ & $3.56(3.8)$ & $0.588(0.08)$ \\
Low stake winning & $3.36(2.0)$ & $2.59(1.6)$ & $0.619(0.08)$ \\
High stake winning & $3.35(2.2)$ & $3.72(4.8)$ & $0.595(0.09)$ \\
\hline
\end{tabular}

\section{Response Latency}

Mean ball removal latency did not statistically significantly differ across high stake, low stake and control conditions: $F(1.472,39.735)=2.274, p>0.05$. Furthermore, there were no statistically significant differences in mean ball removal latency, across stake sizes, after winning bets $(F(1.151,32.236)=2.29, p>0.05)$, or after losing bets $(F(2,56)=1.996$, $p>0.05)$.

\section{Probability of Making Correction Predictions (Pcorrect)}

Participants were more likely to make less accurate predictions i.e. predictions with a lower probability of being correct, after betting in the high stake condition than in the low stake and control conditions; and this difference in was observed to be statistically significant $F(2,62)=11.246, p<0.001$, partial $\eta^{2}=0.266$. Post hoc tests identified significant differences between the high stake and control conditions $(p=0.001)$, between the high stake and the low stake conditions $(p=0.012)$ and also between the low stake and control conditions (Fig. 1).

There was a statistically significant difference observed between participants making less accurate predictions of uncertain outcomes after losing high stake bets, after losing lower stake bets and after the control condition $(F(6,62)=8.533, p<0.01$, partial 


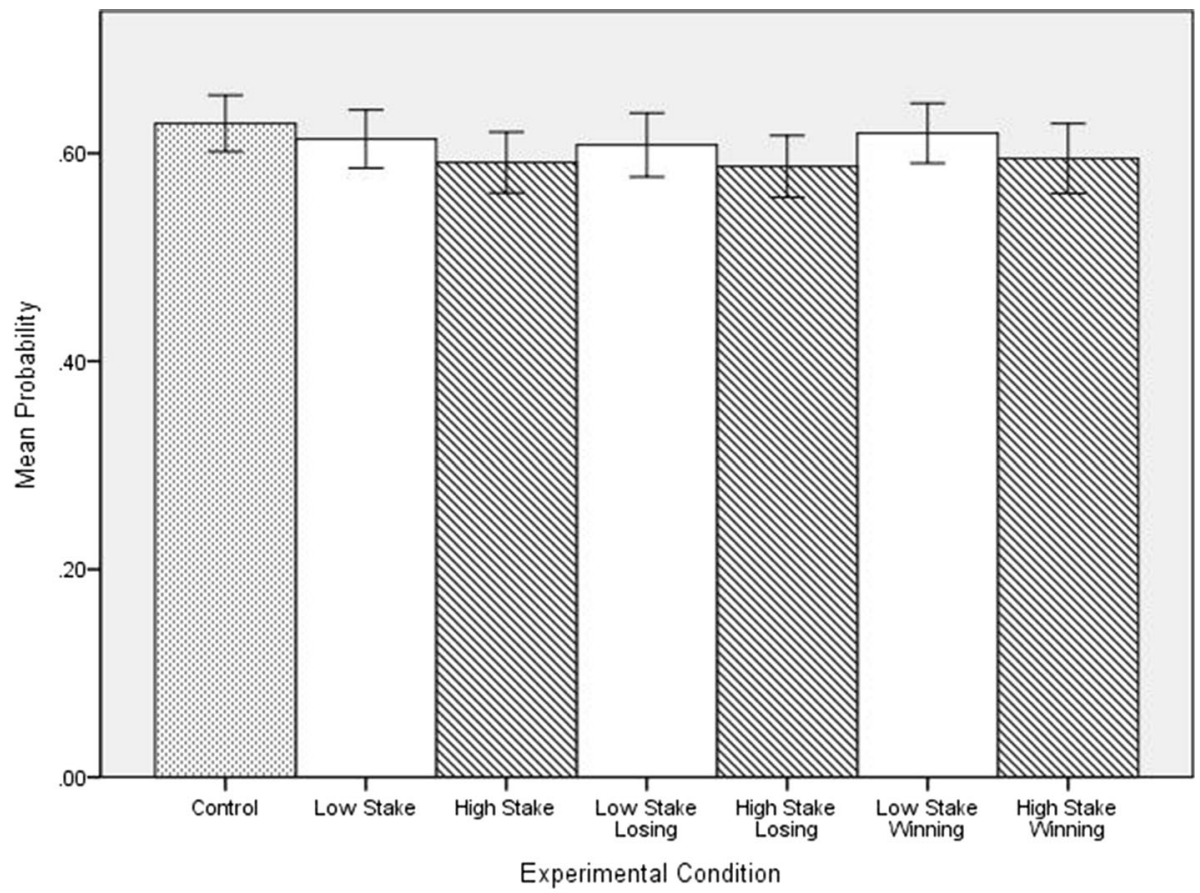

Fig. 1 Mean probability of making accurate predictions (Pcorrect) after betting at different stake sizes and across different betting outcomes

$\eta^{2}=0.216$ ). Post hoc analyses revealed that a statistically significant difference was observed between losing high stake bets and the control condition $(p=0.001)$; with no other statistically significant differences observed.

Participants were more likely to make less accurate predictions about uncertain outcomes after winning high stake bets than after winning lower stake bets and the control condition; and this difference in means was observed to be statistically significant $F(2$, $62)=6.047, p<0.01$ partial $\eta^{2}=0.163$. Post hoc analyses revealed that significant differences were observed between high stake winning and control conditions $(p=0.023)$, and also between the high stake winning and low stake winning conditions $(p=0.042)$. No significant difference was observed between low stake winning and control conditions.

\section{Discussion}

\section{Response Inhibition}

There were no observed statistically significant differences in participants' response inhibition performance after gambling at higher, lower and no monetary stakes. There are a range of possible explanations for the null effect, and the most simplistic interpretation would be that winning and losing at different stake sizes does not subsequently influence one's ability to inhibit prepotent responses. 
However, the lack of impact of stake size on response inhibition performance may be indicative of the inelasticity of inhibitory control of inappropriate but conditioned behavioural responses. Evidence clearly shows that short-term response inhibition performance declines primarily through an increase in working memory load (Hester and Garavan 2005; Stevens et al. 2015). Essentially, it could be the case that the go/no-go test was a significant transition from the gambling task, and that during the transfer into the highly abstract test of response inhibition from the virtual roulette task, the working memory load was reset, allowing the participant to fully attenuate to the task of inhibiting responses. The observed null effect may have been caused because the change in cognitive task required the participant to re-focus attention from the gambling activity to an abstract go/no-go task, enabling the participant to increase preparedness for proactive inhibition. Potentially, if a measure of response inhibition was embedded in the virtual roulette gambling task, one may have observed a decline in performance because of increased cognitive load in response to the incurred monetary wins and losses.

Alternatively, the lack of effect of winning and losing at various stake sizes on response inhibition may be indicative that response inhibition deficits observed in problem gambling populations are a result of either a pre-existing vulnerability in ventromedial prefrontal cortex (vmPFC) functioning, or a result of extensive, repetitive gambling exposure. Although participants in the study were self-reported frequent gamblers, the participants reported that they did not gamble to an extent that had negative consequences beyond moderate monetary loss. Therefore, it is possible that the response inhibition deficit observed in problem gamblers is a developmental consequence of gambling excessively over an extended period of time.

A further possible explanation for the null effect observed on response inhibition is that the stake size parameters used within the experimental design were not large or disparate enough to demonstrate a possible effect. Fundamentally, it is possible that at larger stakes, for example, $£ 50$ or even $£ 100$ per bet that an impact, positive or negative, on response inhibition may have been observed. Ultimately, the potential effect of stake sizes higher than $£ 20$ per bet, and across different research populations, must be researched before any firm conclusions can be made regarding the null effect of stake size on response inhibition.

\section{Reflection Impulsivity}

After gambling at higher stakes, in comparison to lower stakes, participants' quality of decision-making reduced, in terms of accurately predicting which ball colour was in the majority. Furthermore, this finding could not be explained based on participants in the high stake conditions not sampling enough information from the urn, or using less time to evaluate the information, because there was no statistically significant difference observed between the high stake and low stake gambling conditions for these dependent variables.

Unlike the go/no-go task, the adapted information sampling task was relatively analogous to a gambling context, in the sense that the participant was required to interpret imperfect information under uncertainty, with an opportunity to win a monetary prize based on accurate predictions. The gambling context of the measure of reflection impulsivity may account for the observed effect of gambling at higher and lower stakes (incurring larger and smaller wins and losses) affecting this specific executive function performance in the short-term, in contrast to response inhibition. Specifically, the effect of winning and losing at different stake sizes when gambling is more likely to impact executive functions relevant to cognitive control in other similar gambling domains rather than in non-gambling measures of executive function. 
The reduction in the quality of decision-making after gambling at higher stakes in comparison to lower stakes was observed in participants when both winning and losing; suggesting that the reduction cannot be explained based on severity of negative affective states resulting from incurring larger monetary losses. In the problem gambling literature it is evident that when exposed to gambling-related stimuli, problem gamblers produce an increased responsiveness in fronto-striatal reward processing and increased attentional focus onto the gambling stimuli, in comparison to non-gamblers (Goudriaan et al. 2014). Goudriaan et al. (2014), in further review of the neuroimaging literature, concluded that when presented with gambling cues, problem gamblers experience anticipation of engaging in gambling, similar to craving. With respect to the non-problem gamblers in the current study, the experience of gambling wins and losses may have created an enhanced incentive salience for further potential monetary gain in the information sampling task, in terms of a cue-reactivity response. Ultimately, enhanced reward salience stimulated by the presence of gambling stimuli may affect cognitive control in gambling behaviour (Goudriaan et al. 2014). The reduction in making optimal predictions based on evaluating available information to calculate probability, and therefore the most advantageous response, could be explained as participant hyper-reactivity to gambling cues in the adapted information sampling task as a consequence of experiencing more arousing wins and losses in the high stake gambling condition.

\section{Implications}

The observation that participants' quality of decision-making is reduced when gambling at higher stakes is theoretically significant from several perspectives. Fundamentally, it provides support for the proposition that structural characteristics of gambling activities can impact executive functioning relevant to cognitive control in gambling contexts. The vast majority of the literature in problem gambling assumes that deficits in executive functioning in addiction populations are a result of pre-existing neurological vulnerabilities. However, the explorative findings of this experimental investigation suggest that gambling activities where participants can choose to gamble at disparate stake sizes may facilitate impairment in reflection impulsivity, at least in the short-term.

The observation of impairment in decision-making after gambling at higher stakes, independent of winning or losing such bets, indicates that stake size as a structural characteristic is not solely relevant when considering the impact of rate of monetary loss on gambling behaviour but may also reduce cognitive control when gambling. Gambling at higher stakes may affect arousal levels to an extent that hyper-reactivity to potential rewards is created as a result of enhanced reward salience. Hyper-reactivity to gambling related cues may influence attentional processes applied in the evaluation of relevant probability information to make accurate assessment of available information and subsequently, advantageous behavioural decisions. This impaired cognitive response to higher stake gambling via increased cue-reactivity, theoretically may also precipitate a diminished sensitivity to potential monetary losses (Goudriaan et al. 2014). Escalation of monetary wins and losses in a gambling context appears to affect reward and punishment sensitivity in subsequent gambling activity, which in turn, may begin to account for within-session loss-chasing despite the probability of exacerbating the negative consequences of gambling.

Although clearly indicating the potential for structural characteristics to affect executive functioning in gambling contexts, it is important not to make excessive conclusions regarding the implications of the findings at this preliminary stage. Essentially, after 
substantial replication of the current experimental findings, further investigation is required to identify the neurological mechanism that underlies the observed cognitive impairment at higher staking levels. Moreover, before concluding that gambling at various stake sizes does not affect post-gambling response inhibition performance, it is prudent to assess response inhibition specifically within a task that is analogous to gambling, to control for any impact on cognitive load that is likely to affect response inhibition.

\section{Limitations}

Although this study is one of the few existing experimental gambling studies to use real monetary values, it must be noted that the task, although analogous to real gambling contexts, was not a direct replication of gambling activities. Not only was the gambling behaviour devoid of physical and environmental characteristics that influence gambling behaviour, but the money used in the experiment was provided by the experimenter and therefore limited the potential negative consequences of losing bets. Negative consequences remained when participants lost monetary sums because if the bet was not lost they could have retained the value of that stake at the end of the gambling task for personal gain. However, it is probable that the severity of negative consequences was limited to an extent, given that the monetary losses did not stem from the participants' personal resources.

A limitation of the reflection impulsivity findings specifically was the latency of the information sampling task in relation to the independent variable i.e. betting outcomes at various stake sizes. In summary, the participants completed the measure of reflection impulsivity at the end of each of the five experimental conditions rather than immediately after the result of the bets. Given that the only variable to be manipulated in each condition was the stake size and betting outcome, it is still possible to conclude with confidence that the difference in reflection impulsivity performance is attributable to stake size. However, the target of future research should seek to simplify the complexity of the research design and measure the impact on reflection impulsivity of gambling at various stake sizes in a variety of latencies post-gambling.

Finally, it must be acknowledged that at this stage there are limitations to the extent to which the findings can be generalised to wider gambling populations. Although the sample was deliberately skewed towards having a higher proportion of male participants, in order to be reflective of the demographics of regular high stake gambling machine users in Great Britain (Wardle et al. 2010), it is important in future research to explore potential sex differences in any effects or null effects observed within the current study. Fundamentally, because of the demands of experimental measurements there are limitations on the potential sample size and the level of randomness in the sampling procedure. Therefore, it is essential that the observed statistically significant effects (and null effects) within this study are replicated in future studies that use more representative and geographically diverse samples, before any conclusions can be generalised confidently to wider gambling populations.

\section{Conclusion}

The chasing of losses during a gambling session by betting larger and varied amounts despite the accumulation of significant losses is a central risk factor for problem gambling, yet loss chasing is not very well understood (Campbell-Meiklejohn et al. 2008). The 
objective of this study was to begin to understand potential explanatory factors that could account for loss of cognitive control that may precipitate within-session loss-chasing. This study investigated the impact of winning and losing at higher and lower stakes sizes, on two primary executive processes that are central to loss of cognitive control, namely response inhibition and reflection impulsivity. Although stake size did not appear to affect post-gambling inhibitory processes in participants, further research is required to replicate this finding and conclude this with confidence. However, gamblers were observed to have higher reflection impulsivity when gambling at higher stakes in contrast to lower stakes. This suggests that the opportunity for participants to substantially increase stake size on a gambling activity may be a risk factor for impaired cognitive performance when gambling, and perhaps create vulnerability for within-session loss-chasing in some players. Before this study can be used to inform regulatory policy regarding staking regulation in gambling activities, with the aim to reduce gambling-related harm, substantial replication of this finding within in vivo gambling settings is required.

\section{References}

American Psychiatric Association. (2000). Diagnostic and statistical manual of mental disorders (4th ed., text rev.).doi:10.1176/appi.books.9780890423349

Barkley, R. A. (1997). Behavioural inhibition, sustained attention and executive functions: constructing a unifying theory of ADHD. Psychological Bulletin, 121, 65-94. doi:10.1037/0033-2909.121.1.65.

Belin, D., Mar, A. C., Dalley, J. W., Robbins, T. W., \& Everitt, B. J. (2008). High impulsivity predicts the switch to compulsive cocaine-taking. Science, 320, 1352-1355. doi:10.1126/science.1158136.

Billieux, J., Lagrange, G., Van der Linden, M., Lançon, C., Adida, M., \& Jeanningros, R. (2012). Investigation of impulsivity in a sample of treatment-seeking pathological gamblers: A multidimensional perspective. Psychiatry Research, 198(2), 291-296. doi:10.1016/j.psychres.2012.01.001.

Blaszczynski, A. (2013). A critical examination of the link between gaming machines and gambling related harm. Journal of Gambling Business and Economics, 7(3), 55-76. doi:10.5750/jgbe.v7i3.818.

Blaszczynski, A., \& Nower, L. (2002). A pathways model of problem and pathological gambling. Addiction, 97(5), 487-499.

Block, J., Block, J. H., \& Harrington, D. M. (1974). Some misgivings about the matching familiar figures test as a measure of reflection impulsivity. Developmental Psychology, 10, 611-632.

Braverman, J., \& Shaffer, H. (2010). How do gamblers start gambling: Identifying behavioural markers for high-risk internet gambling. European Journal of Public Health, 22(2), 273-278. doi:10.1093/eurpub/ ckp232.

Breen, R. B., \& Zuckerman, M. (1999). Chasing in gambling behavior: Personality and cognitive determinants. Personality and Individual Differences, 92, 1097-1111.

Campbell-Meiklejohn, D. K., Woolrich, M. W., Passingham, R. E., \& Rogers, R. D. (2008). Knowing when to stop: The brain mechanisms of chasing losses. Biological Psychiatry, 63, 293-300. doi:10.1016/j. biopsych.2007.05.014.

Clark, L., Crooks, B., Clarke, R., Aitken, M. R. F., \& Dunn, B. D. (2012). Physiological responses to nearmiss outcomes and personal control during simulated gambling. Journal of Gambling Studies, 28(1), 123-137. doi:10.1007/s10899-011-9247-z.

Clark, L., Lawrence, A., Astley-Jones, F., \& Gray, N. (2009a). Gambling near-misses enhance motivation to gamble and recruit win-related brain circuitry. Neuron, 61(3), 481-490. doi:10.1016/j.neuron.2008.12. 031 .

Clark, L., Robbins, T. W., Ersche, K. D., \& Sahakian, B. J. (2006). Reflection impulsivity in current and former substance users. Biological Psychiatry, 60, 515-522. doi:10.1016/j.biopsych.2005.11.007.

Clark, L., Roiser, J. P., Robbins, T. W., \& Sahakian, B. J. (2009b). Disrupted reflection impulsivity in cannabis users but not current or former ecstasy users. Journal of Psychopharmacology, 23, 14-22. doi:10.1177/0269881108089587.

Coates, E., \& Blaszczynski, A. (2013). Predictors of return rate discrimination in slot machine play. Journal of Gambling Studies, 29(4), 631-645. doi:10.1007/s10899-013-9375-8. 
Cohen, L. J., Nesci, C., Steinfel, M., Haeri, S., \& Galynker, I. (2010). Investigating the relationship between sexual and chemical addictions by comparing executive function in subjects with pedophilia or opiate addiction and healthy controls. Journal of Psychiatry Practice, 16, 405-412. doi:10.1097/01.pra. $0000390759.04581 .7 \mathrm{c}$

Conversano, C., Marazziti, D., Carmassi, C., Baldini, S., Barnabei, G., \& Dell'Osso, L. (2012). Pathological gambling: a systematic review of biochemical, neuroimaging and neuropsychological findings. Harvard Review of Psychiatry, 20, 130-148. doi:10.3109/10673229.2012.694318.

Corless, T., \& Dickerson, M. (1989). Gamblers' self-perceptions of the determinants of impaired control. British Journal of Addiction, 84, 1527-1537.

Delfabbro, P. H., Falzon, K., \& Ingram, T. (2005). The effects of parameter variations in electronic gambling simulations: Results of a laboratory-based pilot study. Gambling Research, 17, 7-25. doi:10. 1002/9781118316078.ch4.

Derryberry, D., \& Reed, M. A. (2002). Anxiety-related attentional biases and their regulation by attentional control. Journal of Abnormal Psychology, 111, 225-236.

Dickerson, M., Hinchy, J., \& Fabre, J. (1987). Chasing, arousal and sensation seeking in off-course gamblers. British Journal of Addiction, 82, 673-680.

Dixon, M. R., Maclin, O. H., \& Daugherty, D. (2006). An evaluation of response allocations to concurrently available slot machine simulations. Behavior Research Methods, 38(2), 232-236. doi:10.1037/ a0023630.

Ferris, J., \& Wynne, H. (2001). The Canadian problem gambling index: Final report. Submitted for the Canadian Centre on Substance Abuse.

Fillmore, M. T. (2003). Drug abuse as a problem of impaired control: Current approaches and findings. Behavioral \& Cognitive Neuroscience Reviews, 2, 179-197. doi:10.1177/1534582303257007.

Goldstein, R. Z., \& Volkow, N. D. (2002). Drug addiction and its underlying neurobiological basis: Neuroimaging evidence for the involvement of the frontal cortex. American Journal of Psychiatry, 159(10), 1642-1652. doi:10.1176/appi.ajp.159.10.1642.

Goudriaan, A. E., Oosterlaan, J., deBeurs, E., \& van den Brink, W. (2006). Neurocognitive functions in pathological gambling: A comparison with alcohol dependence, Tourette syndrome and normal controls. Addiction, 101, 534-547. doi:10.1111/j.1360-0443.2006.01380.x.

Goudriaan, A. E., Yucel, M., \& van Holst, R. J. (2014). Getting a grip on problem gambling: What can neuroscience tell us? Frontiers in Behavioural Neuroscience, 8, 141. doi:10.3389/fnbeh.2014.00141.

Hester, R., \& Garavan, H. (2005). Working memory and executive function: the influence of content and load on the control of attention. Memory \& Cognition, 33, 221-233. doi:10.3758/BF03195311.

Holmes, E. A., Craske, M. G., \& Graybiel, A. M. (2014). Psychological treatments: A call for mental-health science. Nature, 511, 287-289. doi:10.1038/511287a.

Kertzman, S., Lowengrub, K., Aizer, A., Vainder, M., Kotler, M., \& Dannon, P. (2008). Go-no-go performance in pathological gamblers. Psychiatry Research, 161, 1-10. doi:10.1016/j.psychres.2007.06. 026.

Kräplin, A., Dshemuchadse, M., Behrendt, S., Scherbaum, S., Goschke, T., \& Bühringer, G. (2014). Dysfunctional decision-making in pathological gambling: Pattern specificity and the role of impulsivity. Psychiatry Research, 215(3), 675-682. doi:10.1016/j.psychres.2013.12.041.

Lawrence, A. J., Luty, J., Bogdan, N. A., Sahakian, B. J., \& Clark, L. (2009a). Impulsivity and response inhibition in alcohol dependence and problem gambling. Psychopharmacology (Berl), 207(1), 163-172. doi:10.1007/s00213-009-1645-x.

Lawrence, A. J., Luty, J., Bogdan, N. A., Sahakian, B. J., \& Clark, L. (2009b). Problem gamblers share deficits in impulsive decision-making with alcohol-dependent individuals. Addiction, 104(6), 1006-1015. doi:10.1111/j.1360-0443.2009.02533.x.

Lesiuer, H. R. (1979). The compulsive gambler's spiral of options and involvement. Psychiatry, 42, 79-87.

Livingstone, C., Wooley, R., Zazryn, T., Bakacs, L., \& Shami, R. (2008). The relevance and role of gaming machine games and game features on the play of problem gamblers. In Report for the Independent Gambling Authority (IGA). Adelaide: South Australia Independent Gambling Authority. http://www. iga.sa.gov.au/pdf/0801/Final\%20report.Print.Feb08.pdf

O'Connor, J., \& Dickerson, M. (2003). Definition and measurement of chasing in off-course betting and gaming machine play. Journal of Gambling Studies, 19, 359-386. doi:10.1046/j.1360-0443.2003. 00232.x.

Parke, J., \& Parke, A. (2013). Does size really matter? A review of the role of stake and prize levels in relation to gambling related harm. Journal of Gambling Business and Economics, 7(3), 77-110. doi:10. 5750/jgbe.v7i3.819.

Potenza, M. N. (2014). The neural bases of cognitive processes in gambling disorder. Trends in Cognitive Sciences, 18(8), 429-438. doi:10.1016/j.tics.2014.03.007. 
Quednow, B. B., Kuhn, K. U., Hoppe, C., Westheide, J., Maier, W., Daum, I., \& Wagner, M. (2007). Elevated impulsivity and impaired decision-making cognition in heavy users of MDMA. Psychopharmacology (Berl), 189, 517-530. doi:10.1007/s00213-005-0256-4.

Sharpe, L. (2002). A reformulated cognitive behavioral model of problem gambling. A biopsychosocial perspective. Clinical Psychology Review, 22, 1-25. doi:10.1016/S0272-7358(00)00087-8.

Sharpe, L., Walker, M., Coughlan, M. J., Enersen, K., \& Blaszczynski, A. (2005). Structural changes to electronic gaming machines as effective harm minimization strategies for non-problem and problem gamblers. Journal of Gambling Studies, 21(4), 503-520. doi:10.1007/s10899-005-5560-8.

Spada, M. A., \& Roarty, A. (2015). The relative contribution of metacognitions and attentional control to the severity of gambling in problem gamblers. Addictive Behaviors Reports, 1, 7-11. doi:10.1016/j.abrep. 2015.02.001.

Stevens, T., Brevers, D., Chambers, C. D., Lavric, A., McLaren, I. P. L., Mertens, M., et al. (2015). How does response inhibition influence decision making when gambling? Journal of Experimental Psychology: Applied, 21(1), 15-36. doi:10.1037/xap0000039.

Strakowski, S. M., Fleck, D. E., DelBello, M. P., Adler, C. M., Shear, P. K., McElroy, S. L., et al. (2009). Characterising impulsivity in mania. Bipolar Disorders, 11, 41-51. doi:10.1111/j.1399-5618.2008. 00658.x.

van Holst, R. J., van den Brink, W., Veltman, D. J., \& Goudriaan, A. E. (2010). Why gamblers fail to win: a review of cognitive and neuroimaging findings in pathological gambling. Neuroscience \& Biobehavioural Reviews, 34, 87-107. doi:10.1016/j.neubiorev.2009.07.007.

Verdejo-Garcia, A., Lawrence, A. J., \& Clark, L. (2008). Impulsivity as a vulnerability marker for substance-use disorders: Review of findings from high-risk research, problem gamblers and genetic association studies. Neuroscience and Biobehavioral Reviews, 32, 777-810. doi:10.1016/j.neubiorev. 2007.11.003.

Volkow, N. D., Wang, G. J., Fowler, J. S., Tomasi, D., Telang, F., \& Baler, R. (2010). Addiction: Decreased reward sensitivity and increased expectation sensitivity conspire to overwhelm the brain's control circuit. BioEssays, 32(9), 748-755. doi:10.1002/bies.201000042.

Walker, M. B. (1992). The psychology of gambling. Oxford: Pergamon.

Wardle, H., Moody, A., Spence, S., Orford, J., Volberg, R., Jotangia, D., et al. (2010). British gambling prevalence survey 2010. Birmingham: Gambling Commission.

Winstanley, C. A., Eagle, D. M., \& Robbins, T. W. (2006). Behavioural models of impulsivity in relation to ADHD: Translation between clinical and preclinical studies. Clinical Psychology Review, 26, 379-395. doi: 10.1016/j.cpr.2006.01.001

Wright, L., Lipszyc, J., Dupuis, A., Sathees, W. T., \& Schachar, R. (2014). Response inhibition and psychopathology: A meta-analysis of go no go task performance. Journal of Abnormal Psychology, 123(2), 429-439. doi:10.1037/a0036295. 